\title{
Skeletal muscle ultrasound
}

\author{
Sigrid Pillen
}

Radboud University Nijmegen Medical Centre, Donder's Centre of Neuroscience, Department of Neurology, Nijmegen, The Netherlands

\begin{abstract}
Muscle ultrasound is a convenient technique to visualize normal and pathological muscle tissue as it is non-invasive and real-time. Neuromuscular disorders give rise to structural muscle changes that can be visualized with ultrasound: atrophy can be objectified by measuring muscle thickness, while infiltration of fat and fibrous tissue increase muscle echo intensity, i.e. the muscles become whiter on the ultrasound image. Muscle echo intensity need to be quantified to correct for age-related increase in echo intensity and differences between individual muscles. This can be done by gray scale analysis, a method that can be easily applied in daily clinical practice. Using this technique it is possible to detect neuromuscular disorders with predictive values of 90 percent. Only in young children and metabolic myopathies the sensitivity is lower. Ultrasound is a dynamic technique and therefore capable of visualizing normal and pathological muscle movements. Fasciculations can easily be differentiated from other muscle movements. Ultrasound appeared to be even more sensitive in detecting fasciculations compared to EMG and clinical observations, because it can visualize a large muscle area and deeper located muscles. With improving resolution and frame rate it has recently become clear that also smaller scale spontaneous muscle activity such as fibrillations can be detected by ultrasound. This opens the way to a broader use of muscle ultrasound in the diagnosis of peripheral nerve and muscle disorders.
\end{abstract}

Key Words: Skeletal muscle, ultrasound, neuromuscular disorders, quantitative, dynamic

1. Ultrasound of normal muscle tissue

2. Muscle ultrasound in neuromuscular disorders

3. Diagnostic value of muscle ultrasound

4. Muscle ultrasound in specific neuromuscular disorders

5. Dynamic muscle ultrasound and the detection of fibrillations

6. Muscle ultrasound compared to other muscle imaging techniques

\section{Future perspectives in muscle ultrasound}

Ultrasound has been used in medical practice since the early 1950s, when Wild and colleagues discovered the ability of high-frequency ultrasonic waves to visualize living tissues [42]. Since then, the technique of ultrasound has rapidly evolved, leading to its widespread use in almost all fields of medicine because of its non-invasive nature and real-time display. In 1980 it was first discovered that diseased muscles showed a different ultrasound appearance compared to healthy muscles [11]. Next to neuromuscular disorders, malignancies, infections and hematomas and ruptures of the musculoskeletal system can also be detected with ultrasound [7,9,10,24]. Currently, ultrasound is widely available and ultrasound techniques have further improved, resulting in display of muscle tissue with resolutions up to $0.1 \mathrm{~mm}$ [8], which is higher than the resolution that can be achieved with for example 3 Tesla Magnetic Resonance Imaging (MRI), which has a resolution up to $0.2 \times 0.2 \times 1.0 \mathrm{~mm}$ [38]. This article provides insight in the use of muscle ultrasound in the display of healthy and diseased muscle. The use of quantification, dynamic imaging and future directions in these fields are discussed.

\section{Ultrasound of normal muscle tissue}

The sonographic appearance of muscles is fairly distinct and can easily be discriminated from surrounding structures such as subcutaneous fat, bone, nerves, and blood vessels (Fig 1) [18,24]. Normal muscle is relatively black, i.e. has low echo intensity. In the transverse plane, perpendicular to the long axis of the muscle, the muscle has a speckled appearance because of reflections of perimysial connective tissue, which is moderately echogenic (Fig 1). In the longitudinal plane (along the long axis of the muscle) 


\section{Skeletal muscle ultrasound}

European Journal Translational Myology 2010; 1 (4): 145-155
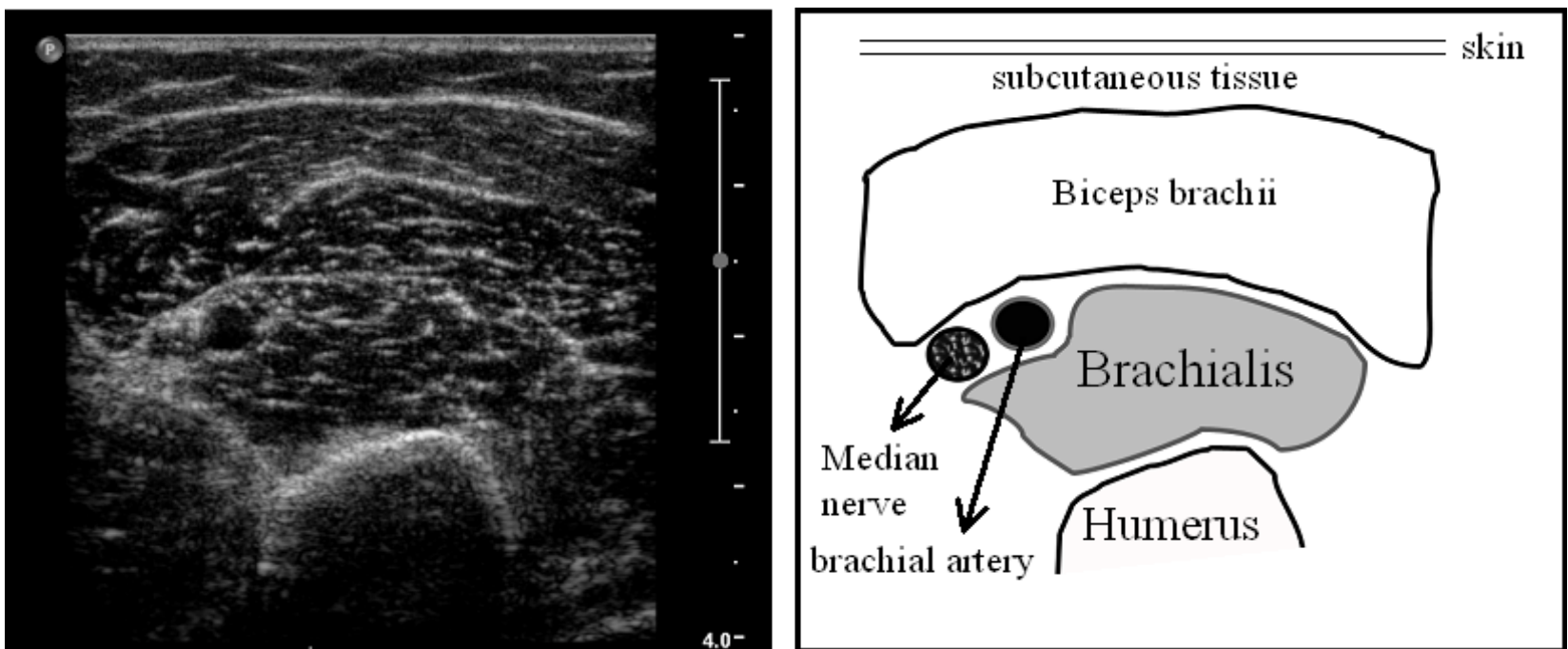

Fig 1. Normal ultrasound measurement of the biceps brachii muscle and surrounding tissues, measured at two thirds of distance from the acromion to the antecubital crease of the left arm. The right panel depicts the different structures schematically.

the fascicular architecture of the muscle becomes visible. Reflections of the perimysial connective tissue

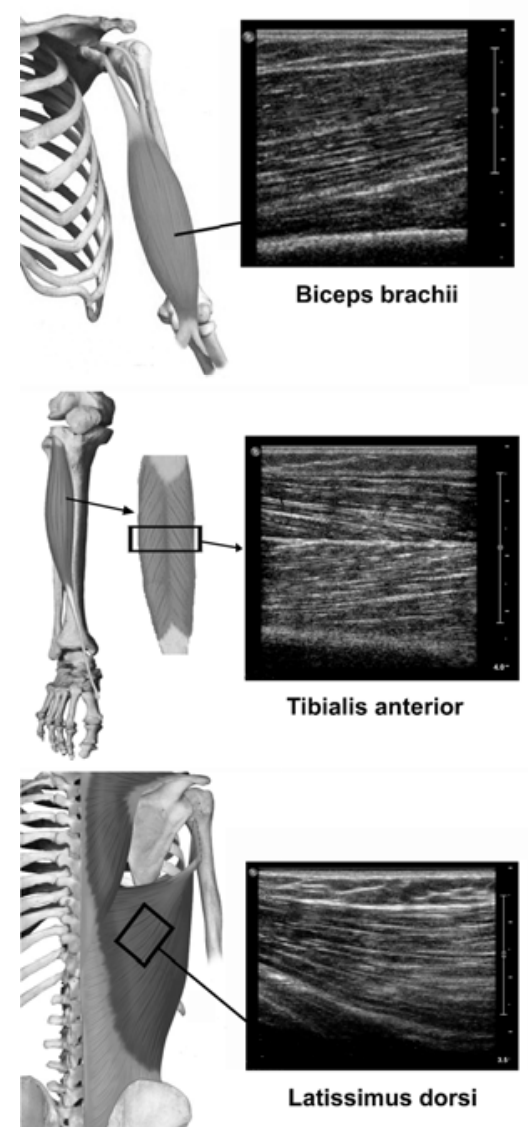

Fig 2. The macro architecture is formed by the way muscle fascicles are organized. They can be parallel as in the biceps brachii, pinnate as in tibialis anterior, or triangular such as in the latissimus dorsi muscle. results in linear, pinnate or triangular structure on the ultrasound image (Fig 2).

The boundaries of the muscle are clearly visible, as the epimysium surrounding the muscle is a highly reflective structure. In normal subjects the bone echo is strong and distinct with an anechoic bone shadow underneath (Fig 3).

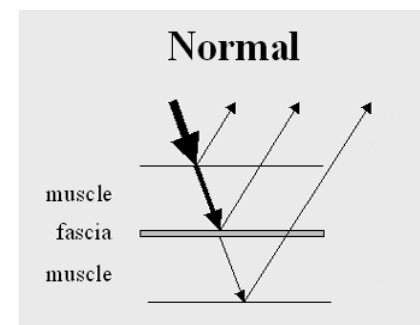

\section{Normal: bone shadow}
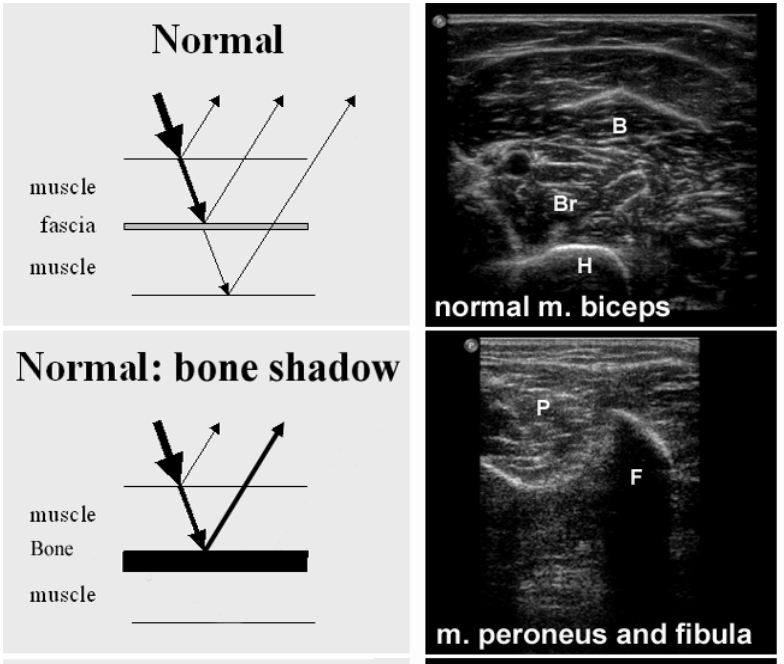

\section{Pathological}
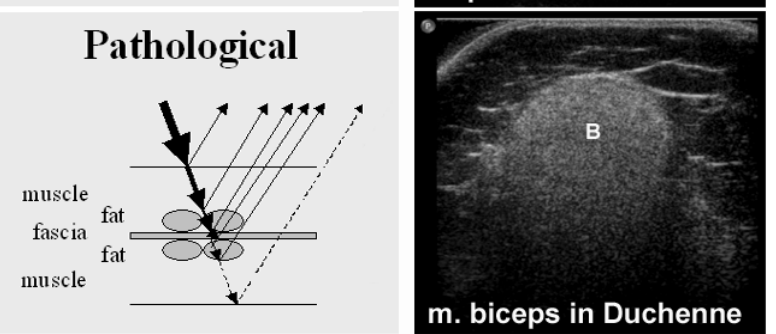

Fig 3. Interactions between ultrasound beam and normal and pathological tissues (left panels) with examples of ultrasound images (right panels). 
Skeletal muscle ultrasound

European Journal Translational Myology 2010; 1 (4): 145-155
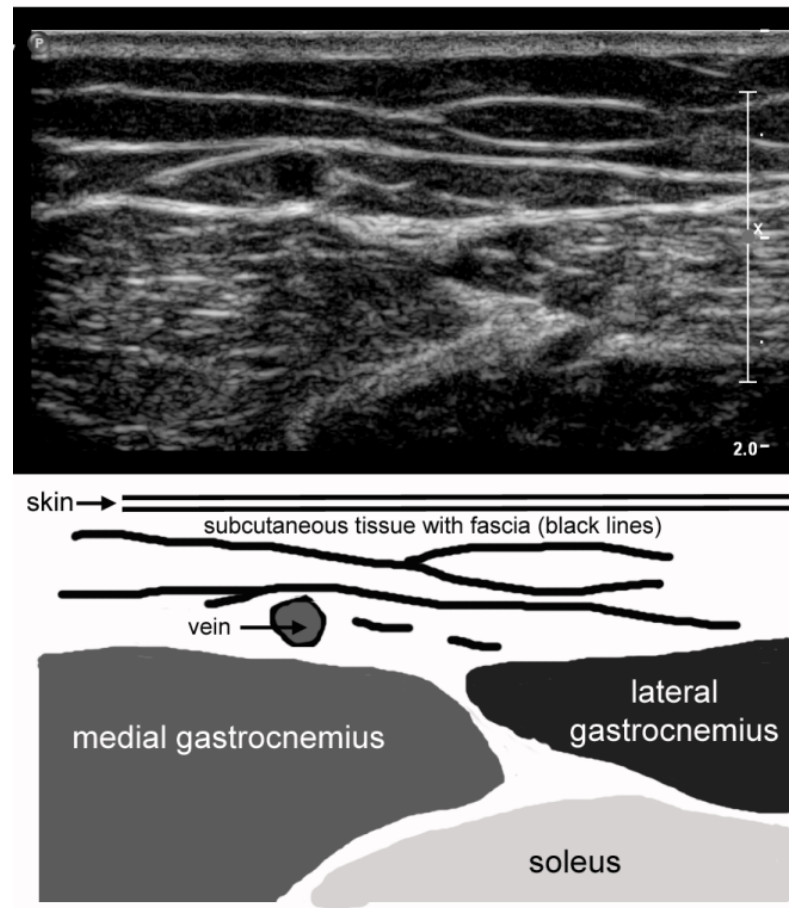

Fig 4. Ultrasound image of the calf showing several layers of fascia in the subcutaneous tissue

Figure 3 shows the interaction between the ultrasound beam and normal and pathological tissues (left panels) with examples of an ultrasound image (right panels). When the ultrasound beam encounters tissue with different acoustical impedance such as the transition from muscle to fascia, a part of the sound is reflected (left upper picture). As healthy muscle contains only little fibrous tissue only a few reflections occur, resulting in a relatively black picture (right upper picture). The acoustical impedance is very different between muscle and bone, causing a strong reflection, with hardly any sound passing through. This results in a bright bone echo with a characteristic bone shadow (middle pictures). In neuromuscular disorders such as Duchenne muscular dystrophy, muscle tissue is replaced by fat and fibrous tissue, resulting in a lot of transitions with different acoustical impedance and much reflection of the ultrasound beam. This explains why the muscle ultrasound image appears white. Additionally attenuation of the ultrasound beam occurs, causing the superficial part of the muscle to be whiter than the deeper part and invisibility of the humerus.

Subcutaneous fat has low echo intensity, but several echogenic septa of connective tissue may be visible within this tissue layer (Fig 4). Nerves and tendons are relatively hyperechoic compared to healthy muscles, whereas blood vessels are hypo- or anechoic circles or lines depending on the direction of the ultrasound beam. When one is unsure about the nature of a round
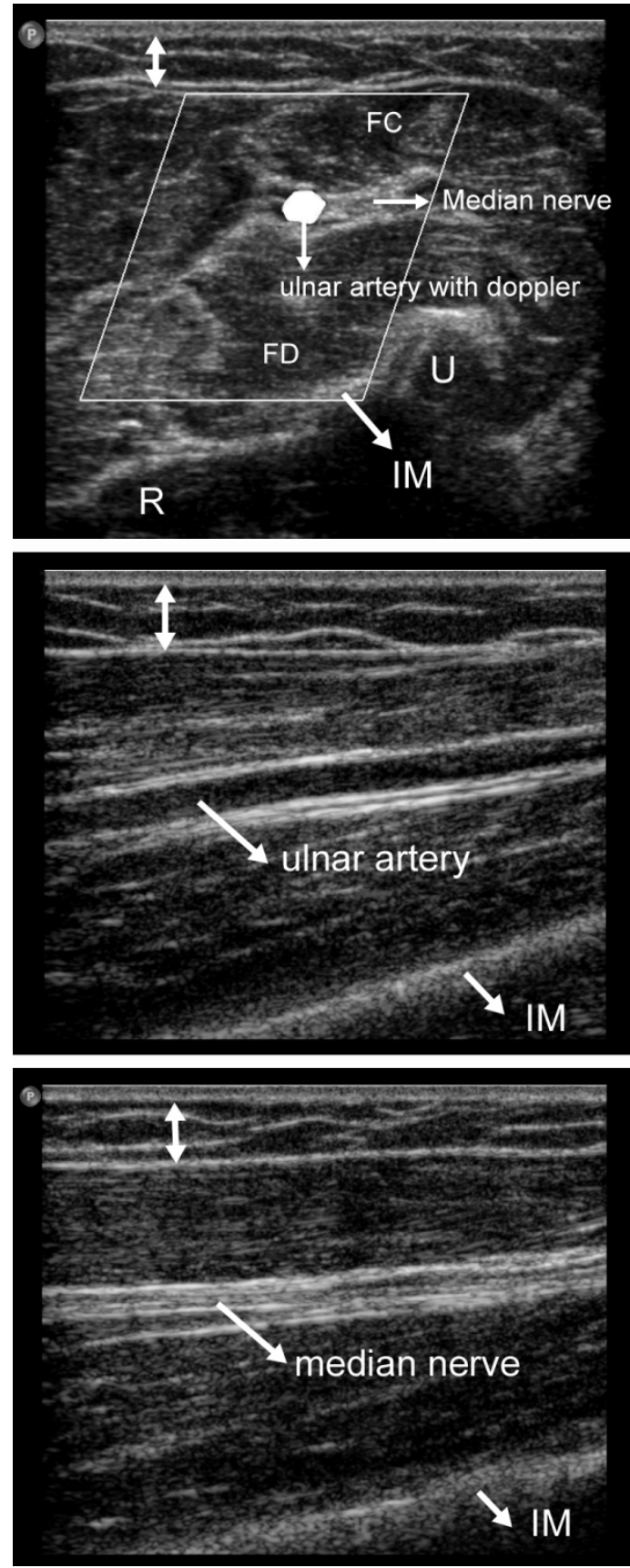

Fig 5. Transverse (upper panel) and longitudinal (middle and lower panel) ultrasound image of the proximal forearm. The median nerve is a hyperechoic circle in the transverse plane with a honeycomb appearance. In the longitudinal plane the fascicle structure of the nerve is visible as hyperechoic lines between the hypoechoic flexor carpi radialis $(F C)$ and flexor digitorum profundus (FD). The ulnar artery is visible as a anechoic line. The transverse image doppler confirms that this structure indeed is an artery. IM = interosseus membrane; double arrow = subcutaneous tissue 


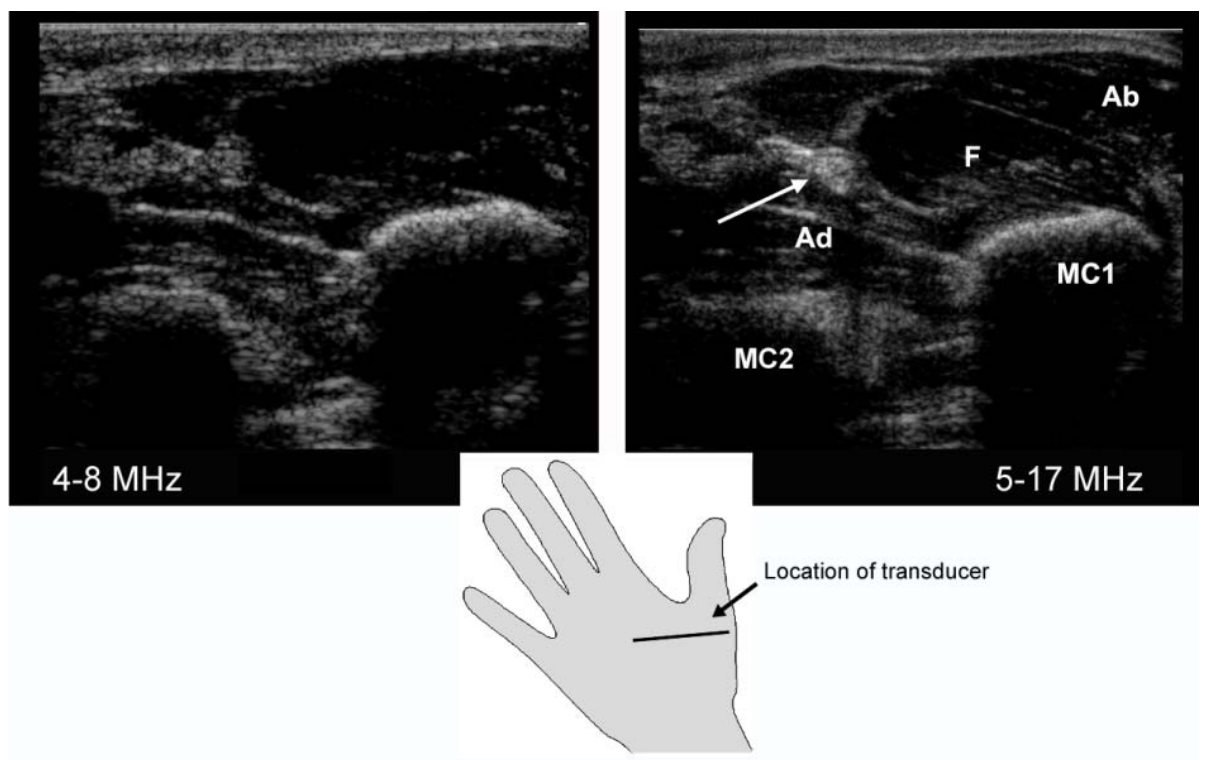

Fig 6. Ultrasound image of small hand muscles. The resolution of the 4-8 MHz transducer is not sufficient to visualize individual hand muscles, whereas in the picture of the 5-17 MHz transducer the m. abductor pollicis brevis $(A b), m$. flexor pollicis brevis (F) and m. adductor pollicis (Ad) can be distinguished. The tendon of the flexor pollicis longus is visible as a bright spot (arrow). MC1 = first metacarpal bone; MC2 = second metacarpal bone.

or linear structure, Doppler imaging can confirm the presence of arteries or veins, by showing blood flow (Fig 5).

All superficial muscles can easily be imaged with ultrasound, although it can be difficult to identify individual small muscles when multiple muscle groups overlap. Recent ultrasound technology using higher frequencies with corresponding higher resolution has made it possible to image individual superficial small muscles in for example the hand (Fig 6). Deeper muscles especially in the pelvic region or around the trunk can be difficult to visualize with sufficient resolution because of the reflection or absorption of sound by superficial tissue layers, such as skin,
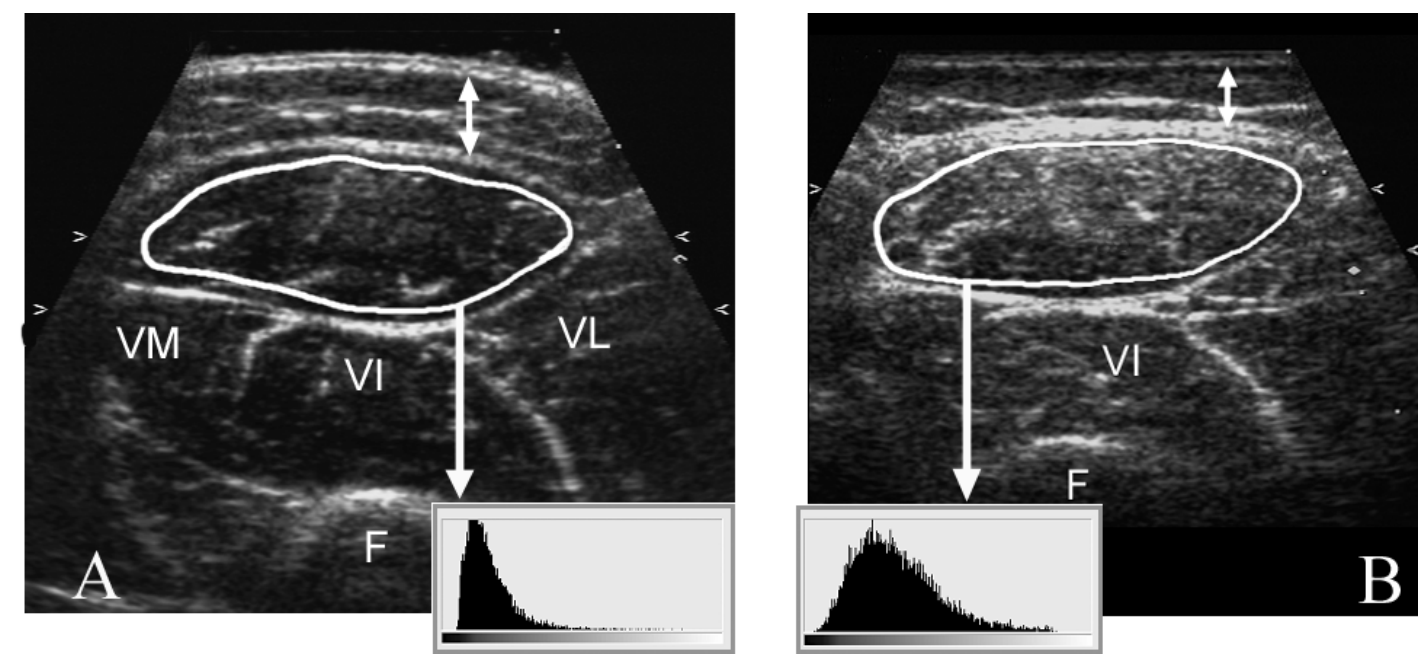

Fig 7. Transverse ultrasound image of a normal left quadriceps muscle (A) and of a patient with Duchenne muscular dystrophy (DMD) (B). Both are 3.5 years of age. The rectus femoris muscle is encircled. Of this region the mean echo intensity is measured, as shown in the histograms below (scale black $=0$; white $=255$ ). The rectus femoris of the DMD patient has increased muscle echo intensity, with the corresponding histogram being displaced to the right. Note the attenuation of the ultrasound beam, i.e. the echo intensity in deeper areas of the muscle is decreased compared to superficial areas. VM, vastus medialis; VL, vastus lateralis; VI, vastus intermedius; F, femur; double arrow, subcutaneous tissue. 
Skeletal muscle ultrasound

European Journal Translational Myology 2010; 1 (4): 145-155

subcutaneous tissue or other muscles.

\section{Muscle ultrasound in neuromuscular disorders}

Previous studies have shown that muscle ultrasound can be of value in the diagnosis of neuromuscular disorders by making it possible to reliably assess muscle thickness and objectify muscle atrophy (or hypertrophy) [12,33,36,37]. Besides muscle atrophy changes in muscle morphology can also be visualized with ultrasound. Neuromuscular disorders can lead to increased muscle echo intensity, i.e. a muscle becomes whiter in appearance $[11,13,36]$. It is thought that the replacement of muscle tissue with fat and fibrosis is the main cause of increased muscle echo intensity, as they increase the number of reflections within the muscle and therefore the mean grey value of the muscle in the ultrasound image (Fig 3) [29,35,37].

Markedly increased muscle echo intensity can easily be detected visually (Fig 7). However, in early stages of a neuromuscular disease or in myopathies that cause only little structural abnormality (such as metabolic myopathies), muscle echo intensity can be only slightly increased and difficult to detect visually. Visual evaluation of muscle ultrasound images has resulted in a sensitivity of only 67 to $81 \%$ for the detection of neuromuscular disorders in children $[13,44]$. These sensitivities are relatively low when muscle ultrasound has to serve the purpose of a screening tool for neuromuscular disorders. Visual assessment is also dependent on the experience of the investigator [17]. Other factors such as systems settings and subjectrelated variables such as age and sex influence muscle echo intensity too [21,35]. Additionally, differences in proportion of fibrous tissue and the orientation of muscle fibres give each muscle a specific appearance on ultrasound, and its own normal range of echo intensities. For example a normal tibialis anterior muscle has a whiter appearance as the rectus femoris muscle (Fig 8). All these factors (observer dependency, influence of system and subject related variables, and differences between muscles) can influence the interrater reliability and variability, and reduce the specificity of the ultrasound findings. The influence of system settings can be eliminated by standardizing the muscle ultrasound protocol and keeping all system settings constant. The other factors, however, cannot be avoided when the ultrasound images are assessed visually. Quantification of muscle echo intensity is more objective and less observer dependent [17], and therefore it is expected to increase the reliability and possibly also the sensitivity of muscle ultrasound. For this reason we chose to develop a technique to quantify muscle echo intensity and assess if quantitative muscle echo intensity is a more reliable and sensitive method to detect neuromuscular disorders.

To quantify muscle echo intensity we used an easily applicable method called quantitative grey scale analysis. This analysis can be performed with a standard histogram function (Fig 7), which is widely available in for example many commercially available computer software programs for image editing, such as Adobe Photoshop (Adobe systems Inc., San Jose, California, USA). After selecting a region of interest within the muscle, the mean grey value of this region can be calculated (automatically) from the histogram (Fig 7). In effect, with quantitative grey scale analysis the entire image is brought back to one value, describing how black, grey or white the muscle in the picture is. As system settings will strongly influence this value, it is very important to keep all settings that influence the grey value such as the gain, compression, focus and TGC constant throughout the measurements. Quantitative grey-scale analysis proved to be better than visual assessment of ultrasound images [25]. Quantification improved the reliability and sensitivity of muscle ultrasound for the detection of neuromuscular disorders compared to visual evaluation alone. Quantification of muscle echo intensity
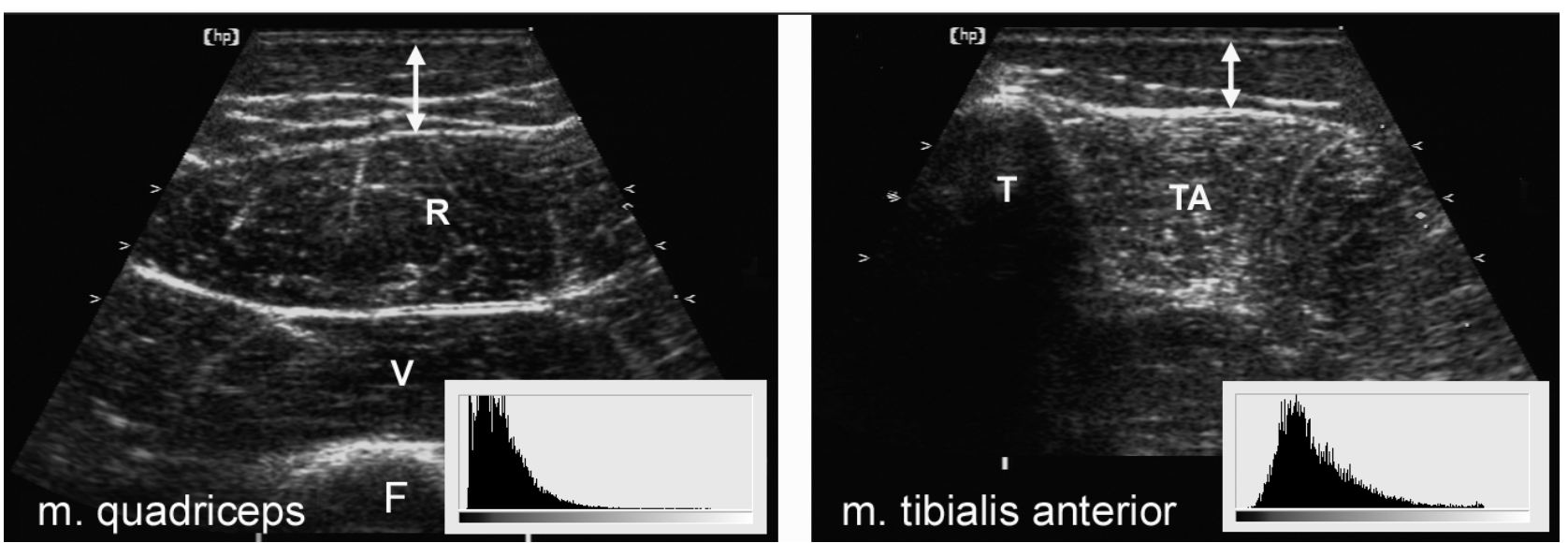

Fig 8. The rectus femoris muscle (left) generally has lower muscle echo intensity than the tibialis anterior muscle (right). Both images are made in the same healthy 13-year-old. Double arrow = subcutaneous tissue; $R=$ rectus femoris muscle; $V=$ vastus intermedius muscle; $F=$ femur; $T A=$ tibialis anterior muscle; $T=$ tibia. 

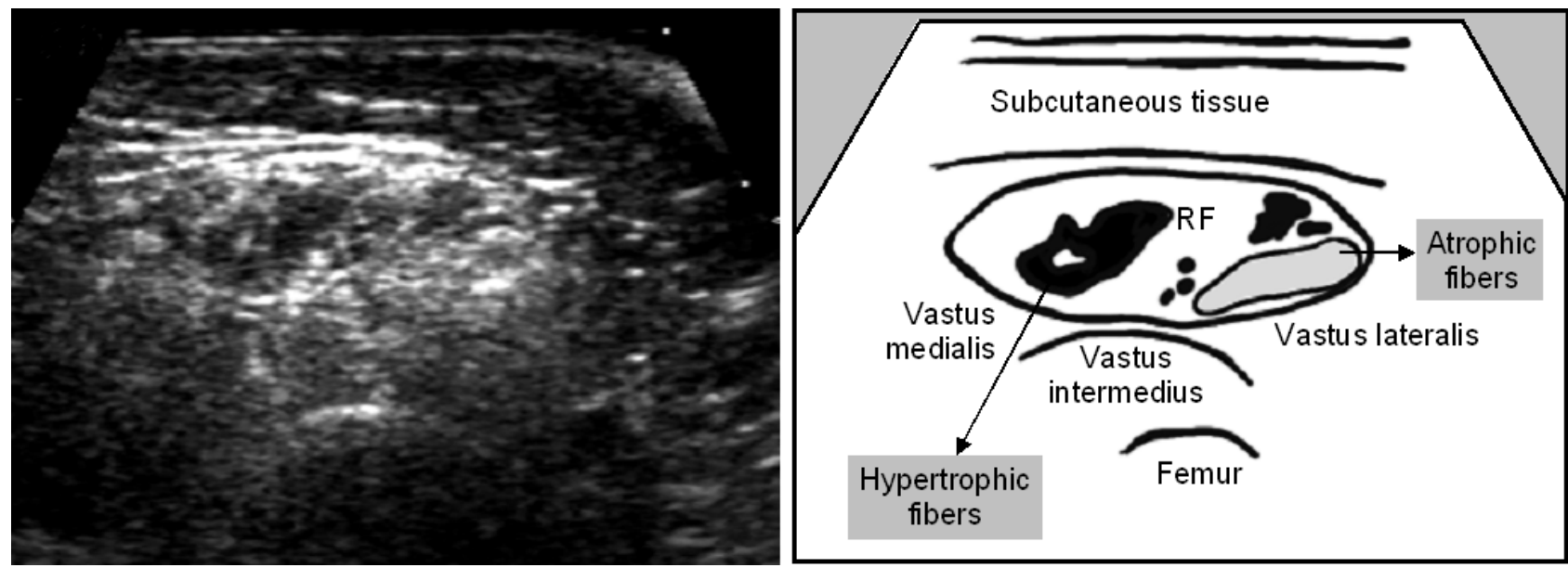

Fig 9. Left quadriceps muscle of a 2-year-old boy with spinal muscular atrophy type 2. The echo intensities are very inhomogenously spread throughout the muscle with a moth-eaten pattern, probably representing atrophic and hypertrophic muscle fibres. Normal structures within and surrounding the muscle are difficult to distinguish. Muscle thickness is diminished. The right panel depicts the different structures schematically. RF = rectus femoris muscle.

therefore has added value in daily clinical practice, even though it is slightly more time consuming (5 to 10 minutes for the examination of 4 muscles) and requires the establishment of normal values.

One of the difficulties of quantitative muscle ultrasound analysis is the application normal values obtained with one ultrasound device to measurements made on another device. This is because each ultrasound device has its own characteristics that are incorporated in the machine and cannot be adjusted (for example the frequency and beam shape of the transducer, or several post-processing techniques, which comprises the processing of the signal between receiving the sound by the transducer and the creation of an image). Copying the user-adjustable system settings to another ultrasound device will therefore not guarantee the same range of muscle echo intensities. This gives rise to the question whether a new set of normal values would have to be gathered for each different ultrasound device used. If this would really be necessary, it would almost certainly influence the chance that quantitative muscle ultrasound will ever be widely applied in daily clinical practice and increase the possibility that it remains a research tool in a research setting only. In a recent study of our group this problem is addressed [30]. It appeared possible to apply a conversion algorithm to reliably use "old" normal values to measurements made on a "new" machine. This means that normal values established with one device can be reliably used on another after establishing and application of this correction factor. It would be advisable to have a dedicated and preservable phantom for this purpose to reliably transfer reference values in case the ultrasound device breaks down.

\section{Diagnostic value of muscle ultrasound}

A prospective pilot study of 33 patients with a suspected neuromuscular disorder followed by a larger study of 150 patients showed that muscle ultrasound is a useful and reliable tool in the diagnostic evaluation of these patients [28,32]. Muscle ultrasound was capable of detecting neuromuscular disorders with a positive predictive value of $91 \%$ and a negative predictive value of $86 \%$. Above the age of 3 years muscle ultrasound was very reliable with a negative predictive value of $95 \%$. Below the age of 3 more false negative results were found (positive predictive value $75 \%$ ). This is caused by the fact that often only few structural changes are present in early stages of a neuromuscular disorder. This result in no or only slightly increased muscle echo intensity. Other false negative ultrasound results were found in patients with a mitochondrial myopathy. This disorder is known for its sparse structural muscle changes, and the effect on muscle ultrasound was confirmed: only 25 to $46 \%$ of the children with a mitochondrial disorder showed a borderline-abnormal or abnormal ultrasound exam [26].

\section{Muscle ultrasound in specific neuromuscular disorders}

The application of muscle ultrasound in specific neuromuscular disorders has also been investigated. The distribution of ultrasound abnormalities throughout the body can aid in the discrimination between neurogenic disorders and myopathies [32]. Neurogenic disorders showed higher echo intensities and more atrophy in the legs than the arms, whereas myopathies showed an equal distribution of pathology between the arms and legs. It was possible to 


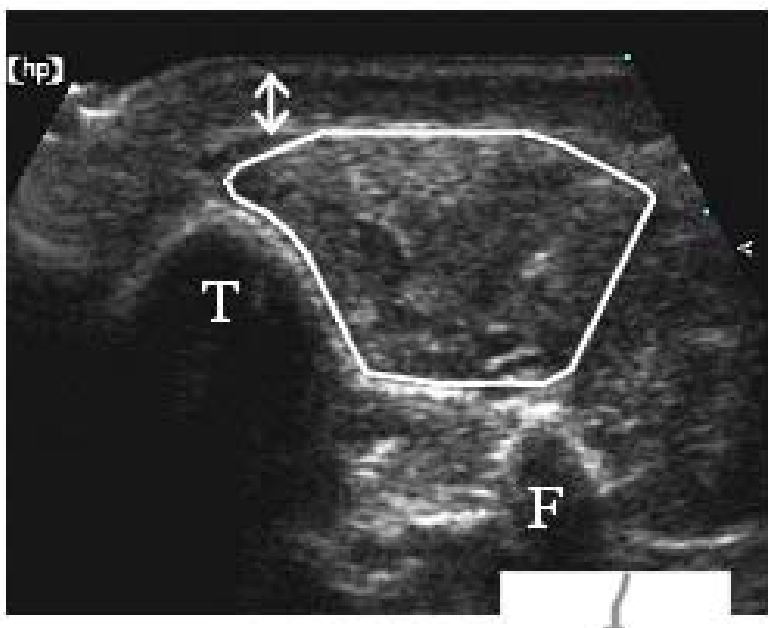

Perpendicular

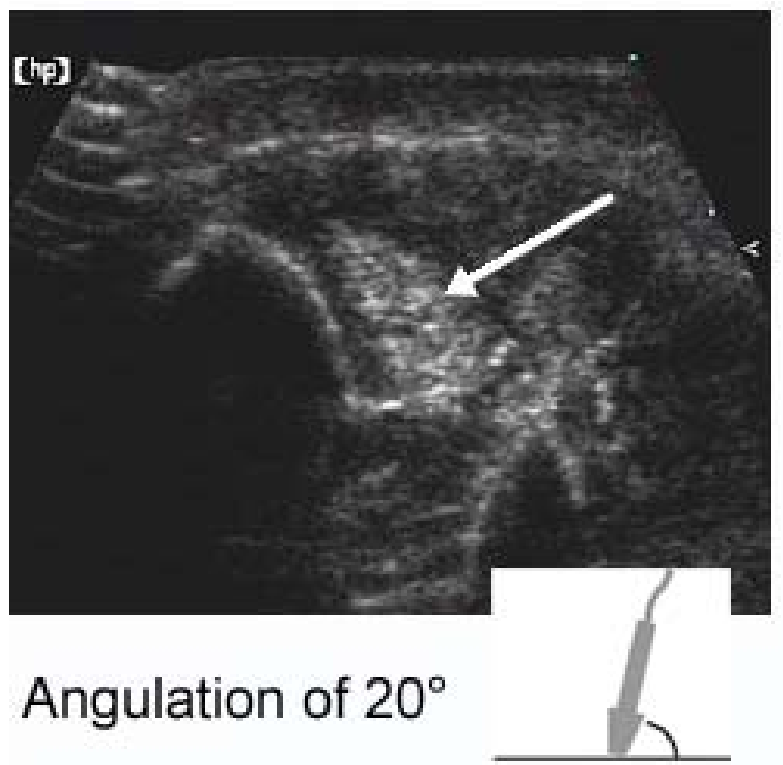

Fig 10. Transverse scan of the anterior tibial muscle at $1 / 4$ between the patella and the lateral malleolus, perpendicular and with an angulation of 20 degrees. The white line depicts the borders of the anterior tibial muscle. The echo intensity of the anterior tibial muscle is increased with a patchy appearance. Angulation of the transducer reveals a hyperechoic part of the anterior tibial muscle (arrow), which was not visible in the perpendicular plane. Double arrow = subcutaneous tissue; $T=$ tibia; $F=$ fibula.

discriminate neurogenic disorders from myopathies with a relatively high positive predictive value of $86 \%$ and a negative predictive value of $84 \%$. Because of the prospective design of this study patients with a false positive ultrasound result, i.e. without a neuromuscular disorder, were included in this study too. Patients with a myopathy showed a similar distribution of pathology compared to those patients. This made it not well possible to discriminate myopathies based on the distribution of pathology throughout the body with sufficiently high predictive values.

Ultrasound findings in specific groups of neuromuscular disorders have not yet been studied prospectively and are until now based on the descriptive literature and expert opinion. Specific neuromuscular disorders differ in the type of muscles that are most affected and in the ultrasonographic appearance of the muscle tissue. For example muscular dystrophies show a homogeneously increased echo intensity (Fig 7), whereas spinal muscular atrophy is accompanied by inhomogeneously, moth-eaten pattern of relatively white and black areas (Fig 9). How well these patterns can aid in the differentiation between specific types of neuromuscular disorders has not yet been investigated. Moreover, these patterns are currently evaluated by visual analysis, which depends on the experience of the observer.

Muscle ultrasound has shown to be helpful in inflammatory myopathies and connective tissue diseases. A patient with juvenile dermatomyositis showed typical ultrasound changes with focally increased echo intensity that became especially apparent after angulation of the transducer (Fig 10) [unpublished data]. A patient with eosinophilic fasciitis demonstrated clearly thickened fascia within and surrounding the muscle, corresponding to subsequent MRI and biopsy findings [31]. Finally muscle ultrasound examination of a patient with morphea profunda revealed extensive muscle involvement, also found at locations where skin lesions were hardly present [39].

\section{Dynamic muscle ultrasound and the detection of fibrillations}

Muscle ultrasound is not only capable of visualizing muscle structure, it is also very suitable to visualize muscle movements. Previous studies have already shown that muscle ultrasound is even more sensitive in detecting fasciculations than EMG [40]. Recent developments in ultrasound resolution and frame rate raised the question whether muscle ultrasound is capable of detecting even smaller pathological muscle movements such as fasciculations. A recent study showed that ultrasound, even though its resolution is still not that of individual muscle fibres, is indeed capable of detecting fibrillations [27]. This finding has important clinical implications, as it is the first technique to non-invasively detect this phenomenon. Moreover, dynamic muscle ultrasound confirms that fibrillations, as electrical phenomena, lead to the actual contraction of muscle fibres. This activity can be 


\section{Skeletal muscle ultrasound}

European Journal Translational Myology 2010; 1 (4): 145-155

abundant in some patients, and the intense and continuous movements of so many muscle fibres, "paradoxically coming alive after denervation", in turn leads to other questions such as the consequences of this activity for metabolism, energy expenditure and (dys-) trophic factor release.

\section{Muscle ultrasound compared to other muscle imaging techniques}

Muscle imaging techniques such as MRI, CT and ultrasound all have their own technical characteristics and advantages. Table 1 summarizes the advantages and disadvantages of each technique. Based on these characteristics, the clinical application of these 3 techniques for muscle imaging in our opinion will be the following:

The main application of muscle ultrasound lies in the screening for the presence of a neuromuscular disorder in general, especially in children or other patients who have difficulties lying still. It can additionally be used to select the optimal site for muscle biopsy, if necessary under direct ultrasound guidance when a muscle is focally affected or when other structures such as vessels and nerves need to be avoided. As the perimysium surrounding muscle fascicles is highly reflective, muscle architecture such as pennation angle or the disruption of the fascicle structure can easily be visualized. Information on muscle architecture can be used to identify lesions caused by trauma such as muscle tears or hematoma [9]. Currently it is not entirely clear what the exact influence of muscle edema on the ultrasound image and muscle echo intensity is. Edema might for example decrease the sensitivity of ultrasound in the early course of inflammatory myopathies, but this has not been investigated.

MRI is very well capable of visualizing both superficial and deeper muscles, which is helpful in determining patterns of muscle involvement. This can aid in identifying the presence of specific congenital myopathies and muscular dystrophies [14,22]. However, prospective studies on the specificity of these finding are not yet available. Muscle MRI is capable of both detecting and differentiating between fatty infiltration and edema. As edema can be an early sign in inflammatory myopathies it is of additional value in case of suspected inflammatory myopathies, even when muscle ultrasound is normal, [34].

CT is also capable of visualizing fatty infiltration, but not fibrosis, in superficial and deep muscles. Unfortunately CT has the disadvantage of ionizing radiation, which makes it an unattractive technique for repeated measurements such as follow-up studies. Moreover, comparative studies between muscle ultrasound and CT have indicated that ultrasound detected early stage structural changes while the CT images were still within the normal range [6]. This is probably because CT cannot distinguish fibrosis from normal muscle tissue and only becomes abnormal in case of fatty infiltration, edema and atrophy. MRI also appears to be more sensitive compared to CT in detecting changes caused by neuromuscular disorders, especially when edema is present [23]. As ultrasound and MRI are good or probably even better alternatives to detect neuromuscular disorders, the clinical application of muscle CT in our opinion is limited when ultrasound or MRI are available. The only application in which CT is preferable is when the extent of intra- or perimuscular calcifications needs to be determined.

Quantification of muscle CT and MRI images is also under investigation. It is possible to quantify muscle CT by measuring Hounsfield units (HU) [1,6]. Hounsfield units range from $-1000 \mathrm{HU}$ (air) to +1000 HU (bone). Fat replacement reduces the average density of muscle because fat has a much lower density (-106 HU) than normal muscle tissue (+54 HU). In this way the extent of fatty infiltration can be objectified. Several parameters of MRI images such as T1 and T2 signal intensities and recovery times can also be quantified. This has been applied to muscle MRI studies in patients with several myopathies and denervation $[15,19]$. The so-called three point Dixon MRI technique is another reliable method to quantify the amount of fat in muscle tissue [16], which has been applied to a follow-up study in patients with Duchenne muscular dystrophy [43]. These quantification methods will improve objectivity of MRI and are considered useful in follow-up studies. But for specific normal values for each muscle and age group are probably needed too, as the fat and fibrosis content of skeletal muscles increases with age and differs between muscles [41]. Normal values could probably further improve the diagnostic accuracy, but are currently not available. Currently quantitative MRI and CT are mainly used in research settings, whereas in daily clinical practice muscle images obtained with these techniques are still evaluated visually.

\section{Future perspectives in muscle ultrasound}

New applications in childhood neuromuscular disease Differentiation based on patterns of muscle involvement

Muscle ultrasound has shown to be capable of describing the distribution of muscle involvement. MRI studies have shown that several congenital myopathies and congenital muscular dystrophies show specific patterns of muscle involvement which can help in the differential diagnosis of these disorders [14]. MRI studies have mainly been confined to leg muscles. Ultrasound is a quick method that could easily visualize the arm muscles too, which can be of additional value as also in the arms selective muscle involvement can be present. New ultrasound developments have resulted in the possibility of making "panoramic"-images, which make it possible to 


\section{Skeletal muscle ultrasound}

European Journal Translational Myology 2010; 1 (4): 145-155

visualize several muscles in one plane. Deeper muscles, however, will remain difficult to display, especially when overlying muscles are affected. To display deeper muscles transducers that can send out lower frequencies have to be used. For an optimal description of any muscle involvement quantification will remain necessary. This implies that future research should also focus on establishment of new normal values for a broad range of also deep and "unusual" muscles

\section{Follow-up and monitoring treatment effect}

Muscle ultrasound can also be used for the follow-up of patients with a neuromuscular disorder. Because of its non-invasiveness, muscle ultrasound can easily be repeated, and this makes it an ideal tool to evaluate new treatment strategies. We are currently studying a cohort of healthy children, following them for 4 years to determine the normal variation in muscle ultrasound with respect to growth, development and muscle force. Additionally, several patients with dermatomyositis, spinal muscular atrophy and duchenne muscular dystrophy are currently included in follow-up studies to determine the value of muscle ultrasound as an outcome measure to evaluate treatment.

\section{Pre-clinical detection of neuromuscular disorders}

Exciting new developments in molecular treatments of neuromuscular disorders have emerged in recent years. There are indications that these treatments will be most effective when started as early as possible, preferably in the presymptomatic stage. This has led to the question if muscle ultrasound can aid in pre-clinical detection of children with neuromuscular disorders. Several neuromuscular disorders such as spinal muscular atrophy and muscular dystrophies already show severely abnormal muscle echo intensities by the time the clinical diagnosis becomes apparent. This would imply that also in a preclinical stadium ultrasound abnormalities will be present. However, the sensitivity of ultrasound is expected to be lower in the preclinical phase as fewer structural changes are present in early stages of the disease. The diagnostic and clinical value of this application of muscle ultrasound could be promising but needs to be further investigated.

Adding nerve ultrasound

Besides visualizing muscles ultrasound is also capable of visualizing nerves. Currently the main clinical focus of nerve ultrasound is the diagnosis of nerve entrapments in adults. It has already been shown that thickened nerves in certain hereditary motor sensory neuropathies are also visible on ultrasound [20]. A combination of nerve and muscle ultrasound in the initial assessment of children with suspected neuromuscular disorders might aid in a further differentiation and the detection of such neuropathies.
New applications outside childhood neuromuscular disease

Muscle ultrasound in adults with neuromuscular disorders

The high diagnostic value of muscle ultrasound in children has prompted investigations of the value of the same technique in adult neuromuscular disease. Several studies are currently performed to investigate if muscle ultrasound can aid in strengthening the diagnosis of amyotrophic lateral sclerosis (ALS) and the differentiation from ALS-mimics [3]. Also, prospective studies on the value of muscle ultrasound in patients with suspected myopathies will soon commence.

Sarcopenia

As people become increasingly older, sarcopenia is a hot item in current research on aging. Muscle ultrasound might aid in the evaluation of muscle structure during aging, as it can visualize changes in muscle structure and muscle thickness $[2,4]$. This can be correlated to muscle function, tendency to fall, effects of treatment etc, to see whether muscle ultrasound could be a useful adjunct in this area too.

Sports medicine

Muscle ultrasound is capable of visualize muscle architecture such as fascicle length and pennation angle. These parameters have already been shown to correlate with force generation and have been used in the evaluation of training in several types of sport [5]. A new application of muscle ultrasound outside the field of neurology might therefore lie in the evaluation of training regimes (force versus duration), effects of overuse, strain and sports injuries.

Bridging function and structure

A very interesting project would be the combination of quantitative dynamic techniques, electrodiagnostic measurements such as surface EMG and force measurements to investigate the interactions between electrical phenomenon, the resulting mechanical (i.e. structural) muscle changes and force generation. This might aid in a further understanding of electromechanical coupling, myofascial force transmission and pathophysiology of disorders in which these interaction are disturbed such as extracellular matrix disorders (for example Ehlers Danlos Syndrome) and myotonic syndromes.

\section{Corresponding Author}

Radboud University Nijmegen Medical Centre, Donder's Centre of Neuroscience, Dept. Neurology, Nijmegen, The Netherlands

E-mail: s.pillen@cukz.umcn.nl 


\section{Skeletal muscle ultrasound}

European Journal Translational Myology 2010; 1 (4): 145-155

\section{References}

[1] Arai Y, Osawa M, Fukuyama Y. Muscle CT scans in preclinical cases of Duchenne and Becker muscular dystrophy. Brain Dev 1995; 17 : 95-103.

[2] Arts IM, Pillen S, Overeem S, Jurgen SH, Zwarts MJ. Rise and fall of skeletal muscle size over the entire life span. J Am Geriatr Soc 2007; 55: 11501152.

[3] Arts IM, van Rooij FG, Overeem S, Pillen S, Janssen HM, Schelhaas HJ, Zwarts MJ. Quantitative muscle ultrasonography in amyotrophic lateral sclerosis. Ultrasound Med Biol. 2008; 34: 354-361.

[4] Arts IM, Pillen S, Schelhaas HJ, Overeem S, Zwarts MJ. Normal values for quantitative muscle ultrasonography in adults. Muscle Nerve. 2010; 41: 32-41

[5] Brancaccio P, Limongelli FM, D'Aponte A, Narici M, Maffulli N. Changes in Skeletal muscle architecture following a cycloergometer test to exhaustion in athletes. J Sci Med Sport 2008 ; 11: 538-41

[6] Cady EB, Gardener JE, Edwards RH. Ultrasonic tissue characterisation of skeletal muscle. Eur J Clin Invest 1983; 13: 469-473.

[7] Campbell SE, Adler R, Sofka CM. Ultrasound of muscle abnormalities. Ultrasound Q 2005; 21: 8794.

[8] Cosgrove D. Ultrasound: general principles. In: Grainger RG, Allison DJ, editors. Diagnostic radiology. Edinburgh: Churchill Livingstone; 1992. pp 65-77.

[9] Fornage BD. The case for ultrasound of muscles and tendons. Semin Musculoskelet Radiol 2000; 4: 375-391.

[10] Hashimoto BE, Kramer DJ, Wiitala L. Application of musculoskeletal sonography. J Clin Ultrasound 1999; 27: 293-299.

[11] Heckmatt JZ, Dubowitz V, Leeman S. Detection of pathological change in dystrophic muscle with B-scan ultrasound imaging. Lancet 1980; 1: 1389-1390.

[12] Heckmatt JZ, Pier N, Dubowitz V. Measurement of quadriceps muscle thickness and subcutaneous tissue thickness in normal children by real-time ultrasound imaging. J Clin Ultrasound 1988; 16: 171-176.

[13] Heckmatt JZ, Pier N, Dubowitz V. Real-time ultrasound imaging of muscles. Muscle Nerve 1988; 11: 56-65.

[14] Jungbluth H, Davis MR, Muller C, Counsell S, Allsop J, Chattopadhyay A, Messina S, Mercuri E, Laing NG, Sewry CA, Bydder G, Muntoni F. Magnetic resonance imaging of muscle in congenital myopathies associated with RYR1 mutations. Neuromuscul Disord 2004; 14: 785790.

[15] Kikuchi Y, Nakamura T, Takayama S, Horiuchi $\mathrm{Y}$, Toyama Y. MR imaging in the diagnosis of denervated and reinnervated skeletal muscles: experimental study in rats. Radiology 2003; 229: 861-867.

[16] Kovanlikaya A, Guclu C, Desai C, Becerra R, Gilsanz V. Fat quantification using three-point dixon technique: in vitro validation. Acad Radiol 2005; 12: 636-639.

[17] Krupinski EA. Computer-aided detection in clinical environment: benefits and challenges for radiologists. Radiology 2004; 231: 7-9.

[18] Lamminen A, Jaaskelainen J, Rapola J, Suramo I. High-frequency ultrasonography of skeletal muscle in children with neuromuscular disease. J Ultrasound Med 1988; 7: 505-509.

[19] Maillard SM, Jones R, Owens C, Pilkington C, Woo P, Wedderburn LR et al. Quantitative assessment of MRI T2 relaxation time of thigh muscles in juvenile dermatomyositis. Rheumatology (Oxford) 2004; 43: 603-608.

[20] Martinoli C, Schenone A, Bianchi S, Mandich P, Caponetto Abbruzzese $M$ et al. Sonography of the median nerve in Charcor-Marie-Tooth disease. AJR Am J Roentgenol 2002; 178: 1553-1556.

[21] Maurits NM, Bollen AE, Windhausen A, De Jager AE, Van Der Hoeven JH. Muscle ultrasound analysis: normal values and differentiation between myopathies and neuropathies. Ultrasound Med Biol 2003; 29: 215-225.

[22] Mercuri E, Jungbluth H, Muntoni F. Muscle imaging in clinical practice: diagnostic value of muscle magnetic resonance imaging in inherited neuromuscular disorders. Curr Opin Neurol 2005; 18: 526-537.

[23] Ozsarlak O, Schepens E, Parizel PM, Van Goethem JW, Vanhoenacker F, De Schepper AM, Martin JJ. Hereditary neuromuscular diseases. Eur J Radiol 2001; 40: 184-197.

[24] Peetrons P. Ultrasound of muscles. Eur Radiol 2002; 12: 35-43.

[25] Pillen S, Keimpema M, Nievelstein RAJ, Verrips A, Kruijsbergen-Raijmann W, Zwarts MJ. Skeletal muscle ultrasonography: visual versus quantitative evaluation. Ultrasound Med Biol 2006; 32: 1315-1321.

[26] Pillen S, Morava E, Van Keimpema M, ter Laak HJ, De Vries MC, Rodenburg RJ, et al. Skeletal muscle ultrasonography in children with a dysfunction in the oxidative phosphorylation system. Neuropediatrics 2006; 37: 142-147. 


\section{Skeletal muscle ultrasound}

European Journal Translational Myology 2010; 1 (4): 145-155

[27] Pillen S, Nienhuis M, van Dijk JP, Arts IM, van Alfen N, Zwarts MJ. Muscles alive: ultrasound detects fibrillations. Clin Neurophysiol. 2009 120: 932-936.

[28] Pillen S, Scholten RR, Zwarts MJ, Verrips A. Quantitative skeletal muscle ultrasonography in children with suspected neuromuscular disease. Muscle Nerve 2003; 27: 699-705.

[29] Pillen S, Tak R, Lammens M, Verrijp K, Arts I, Zwarts M, Van Engelen B, Verrips A. Skeletal muscle ultrasound: correlation between fibrous tissue and echo intensity. Ultrasound Med Biol. 2009; 35: 443-6

[30] Pillen S, Van Dijk JP, Weijers G, Raymann W, De Korte CL, Zwarts MJ. Quantitative grey scale analysis in skeletal muscle ultrasound: a comparison study of two ultrasound devices. Muscle Nerve 2009; 39: 781-786.

[31] Pillen S, van Engelen B, van den Hoogen F, Fiselier T, van der Vossen P, Drost G. Eosinophilic fasciitis in a child mimicking a myopathy. Neuromuscul Disord 2006; 16: 144148.

[32] Pillen S, Verrips A, van Alfen N, Arts IM, Sie LT, Zwarts MJ. Quantitative skeletal muscle ultrasound: Diagnostic value in childhood neuromuscular disease. Neuromuscul Disord 2007; 17: 509-516.

[33] Reeves ND, Maganaris CN, Narici MV. Ultrasonographic assessment of human skeletal muscle size. Eur J Appl Physiol 2004; 91: 116118.

[34] Reimers CD, Finkenstaedt M. Muscle imaging in inflammatory myopathies. Curr Opin Rheumatol 1997; 9: 475-485.

[35] Reimers CD, Fleckenstein JL, Witt TN, MullerFelber W, Pongratz DE. Muscular ultrasound in idiopathic inflammatory myopathies of adults. J Neurol Sci 1993; 116:82-92.

[36] Reimers CD, Schlotter B, Eicke BM, Witt TN. Calf enlargement in neuromuscular diseases: a quantitative ultrasound study in 350 patients and review of the literature. J Neurol Sci 1996; 143: 46-56.

[37] Reimers K, Reimers CD, Wagner S, Paetzke I, Pongratz DE. Skeletal muscle sonography: a correlative study of echogenicity and morphology. J Ultrasound Med 1993; 12: 73-77.

[38] Saupe N, Prüssmann KP, Luechinger R, Bösiger $\mathrm{P}$, Marincek B, Weishaupt D. MR imaging of the wrist: comparison between 1.5- and 3-T MR imaging--preliminary experience. Radiology 2005; 234: 256-64.

[39] Voermans NC, Pillen S, de Jong EM, Creemers MC, Lammens $M$, van Alfen N. Morphea profunda presenting as a neuromuscular mimic. J Clin Neuromuscul Dis 2008; 9: 407-414.

[40] Walker FO, Donofrio PD, Harpold GJ, Ferrell WG. Sonographic imaging of muscle contraction and fasciculations: a correlation with electromyography. Muscle Nerve 1990; 13: 3339.

[41] Wehrli NE, Bural G, Houseni M, Alkhawaldeh K, Alavi A, Torigian DA. Determination of agerelated changes in structure and function of skin, adipose tissue, and skeletal muscle with computed tomography, magnetic resonance imaging, and positron emission tomography. Semin Nucl Med 2007; 37: 195-205.

[42] Wild JJ, Neal D. Use of high-frequency ultrasonic waves for detecting changes of texture in living tissues. Lancet 1951; 1: 655-657.

[43] Wren TA, Bluml S, Tseng-Ong L, Gilsanz V. Three-point technique of fat quantification of muscle tissue as a marker of disease progression in Duchenne muscular dystrophy: preliminary study. AJR Am J Roentgenol 2008; 190: W8-12.

[44] Zuberi SM, Matta N, Nawaz S, Stephenson JB, McWilliam RC, Hollman A. Muscle ultrasound in the assessment of suspected neuromuscular disease in childhood. Neuromuscul Disord 1999; 9: 203-207. 
Skeletal muscle ultrasound

European Journal Translational Myology 2010; 1 (4): 145-155 